\title{
Intramolecular Charge Transfer near a Hydrophobic Surface. 2,6-p-Toluidinonaphthalene Sulfonate in a Reverse Micelle
}

\author{
Debabrata Mandal, Samir Kumar Pal, Anindya Datta and Kankan Bhattacharyya ${ }^{\dagger}$ \\ Department of Physical Chemistry, Indian Association for the Cultivation of Science, \\ Jadavpur, Calcutta 700 032, India
}

\begin{abstract}
Intramolecular charge transfer (ICT) processes of 2,6-p-toluidinonaphthalene sulfonate (TNS) in the water pool of sodium dioctyl sulfosuccinate (AOT) reverse micelle in heptane, is studied using picosecond emission spectroscopy. It is observed that while in aqueous solutions the lifetime $\left(\tau_{\mathrm{f}}\right)$ of TNS is $80 \mathrm{ps}, \tau_{\mathrm{f}}$ increases to $4 \mathrm{~ns}$ in the microemulsion with water to AOT ratio, $w_{0}=4$. With increase in $w_{0}$ as the water pool swells in size, the lifetime and quantum yield of emission decrease and the rate of the ICT process increases. However, the magnitude of the change (at most $\approx 70$ times at $\left.w_{0}=4\right)$ in the rate of the ICT process of TNS compared to ordinary water is smaller than the several thousand fold retardation in the solvation dynamics of water in such a water pool relative to bulk water. This is attributed to the fact that while the solvation dynamics in the water pool is governed by the dielectric relaxation, dynamics of the ICT process is controlled by the static polarity of the medium.
\end{abstract}

Keywords Reverse micelle, ultrafast charge transfer

The behavior of an organic molecule at an interface between two bulk media is often significantly different from that in either of the bulk medium. Since the polarity and viscosity at the interface are often very much different from those of the bulk media, the structure, dynamics and reactivity of an organic or biomolecule at an interface differ markedly from those in the bulk. Interestingly most natural and biological processes occur at such interfaces or confined systems, such as proteins, biomembranes and vesicles. As a result in recent years several new experimental and theoretical techniques have been used to study such systems. Several groups have used surface second harmonic and surface sum frequency generation and picosecond total internal reflection spectroscopy to study the structure and the dynamics at various interfaces. ${ }^{1-3}$ The behavior of water molecules present at such interfaces and perturbed by local electrostatic and hydrogen bond interactions are of special interest because of the unique role played by the water molecules in many biological processes. Recently several groups used the time dependent Stokes shift ${ }^{5-10}$, pulsed NMR and dielectric relaxation techniques ${ }^{11-14}$ to unravel the dynamics of the water molecules in various organized media such as cyclodextrins ${ }^{5,6}$, reverse micelle ${ }^{4,7-9}$ and micelles. ${ }^{10}$ The results of the dynamic Stokes shift studies indicate that while ordinary water molecules relax in the subpicosecond time scale, inside these organized assemblies the solvation dynamics becomes several thousand times slower and occurs in the nanosecond timescale. ${ }^{5-10}$

\footnotetext{
† To whom correspondence should be addressed.
}

In hydrocarbon solvents at concentrations above $\approx 1$ $\mathrm{mM}$ certain surfactants $(e . g$. sodium dioctyl sulfosuccinate, AOT) form so called reverse micelles with average aggregation number 20 and radius $15 \AA$, in which the polar headgroups point inwards., ${ }^{4-9,21-28}$ On addition of water to a solution of AOT in a hydrocarbon solvent a microemulsion is formed which consists of nanometer sized water droplets ("water pool") surrounded by a layer of the surfactant molecule. In heptane the radius $\left(r_{\mathrm{w}}\right)$ of such a water pool is about $2 w_{0}$ (in $\AA$ ) where $w_{0}$ is the number ratio of the water to the surfactant molecules. ${ }^{4,25}$ Such microemulsions are simple yet interesting models of biological membranes and the water molecules confined in biological systems. In such a water pool, the water molecules at the peripheries of the pool are strongly held by the polar or ionic headgroups of the surfactants and are thus "bound", while those at the center of the pool are relatively "free". The amount of free and bound water molecules present in such a microemulsion has been estimated and tabulated by many workers. ${ }^{4,22,23}$ Recently Bright et al. ${ }^{7}$ and our group ${ }^{8,9}$ separately reported that in such a water pool of a microemulsion the solvent relaxation times are about $8 \mathrm{~ns}$ when the pool is small $\left(r_{\mathrm{w}}<10 \AA\right)$ and $2 \mathrm{~ns}$ for bigger water pools. Such nanosecond solvation times are several thousand times slower than the subpicosecond relaxation time in ordinary water reported by Fleming et al. ${ }^{5}$ The photoisomerization of a cyanine dye is also found to be slowed down in such microemulzions. ${ }^{31}$ In the present work we report on the intramolecular charge transfer process of 2,6-p-toluidinonaphthalene sulfonate (TNS) in AOT reverse micelles. We have 
earlier shown that the nonradiative rate of TNS increases exponentially with the polarity parameter $E_{\mathrm{T}}(30)$ of the medium and have assigned this nonradiative process to the polarity dependent twisted intramolecular charge transfer (ICT) process. ${ }^{15-20}$ The main advantage of TNS as a probe for such systems is the fact that it is insoluble in bulk-heptane and because of its charge it stays exclusively in the water pool.

\section{Experimental}

TNS (potassium salt, Aldrich) was used as received. Purification and drying of sodium dioctyl sulfosuccinate (AOT, Aldrich) is described elsewhere. ${ }^{8,9}$ To a solution of AOT in freshly distilled heptane requisite amount of water was added using a microliter syringe to make a microemulsion of $w_{0}=4$. To this solid TNS was added and subsequently more water is added to increase $w_{0}$. For lifetime measurements the sample was excited at $300 \mathrm{~nm}$ using a cavity dumped synchronously pumped dye laser (Coherent 702) pumped by a CW mode locked Nd:YAG laser (Coherent Antares 76S). The fluorescence was detected at magic angle polarization by a Hamamatsu MCP photomultiplier (2809U). ${ }^{8,9}$ Time resolution of this setup is $\approx 50$ ps. Steady state absorption and emission spectra were recorded in JASCO 7850 spectrophotometer and Perkin Elmer MPF 44B spectrofluorimeter respectively.

\section{Results and Discussion}

\section{Steady state spectra}

As mentioned already TNS is insoluble in heptane but dissolves in $0.09 \mathrm{M}$ AOT in heptane in the presence

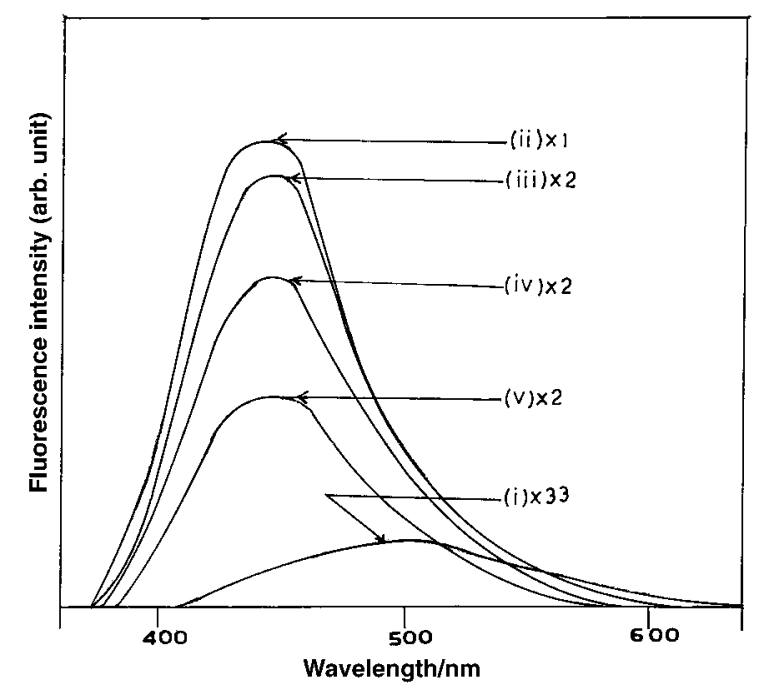

Fig. 1 Emission spectra of TNS, $\lambda_{\mathrm{ex}}=320 \mathrm{~nm}$, (i) in water; (ii) - (v) in heptane with $0.09 \mathrm{M} \mathrm{AOT}$ at $w_{0}=4,8,12$ and 32, respectively.

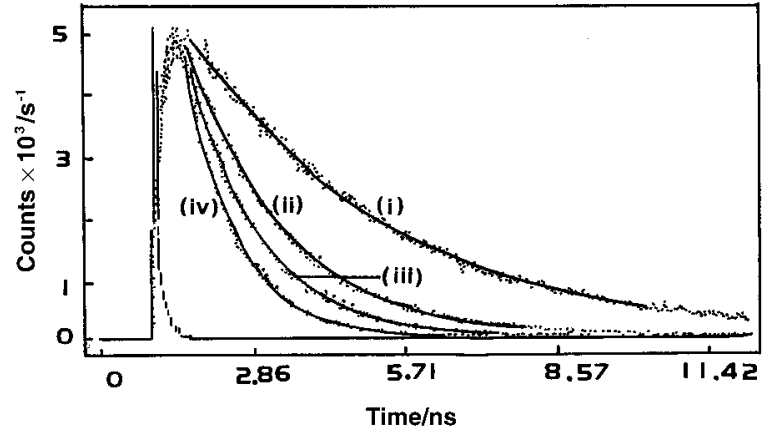

Fig. 2 Fluorescence decay of TNS in 0.09 M AOT in heptane, (i) $w_{0}=4$, (ii) $w_{0}=8$, (iii) $w_{0}=12$ and (iv) $w_{0}=32$. The peak near time zero denotes system response.

of water. In such a microemulsion of $w_{0}=4$ TNS exhibits intense emission with $\lambda_{\max }^{\mathrm{em}}=440 \mathrm{~nm}$ and $\phi_{\mathrm{f}}$ $=0.29$. The $\lambda_{\max }^{\mathrm{em}}$ of TNS in microemulsion is about 40 $\mathrm{nm}$ blue shifted from that of TNS in water $(\approx 480 \mathrm{~nm})$ and also the $\phi_{\mathrm{f}}$ in microemulsion is nearly 290 times higher (Fig. 1). Such dramatic differences of $\lambda_{\max }^{\mathrm{em}}$ and $\phi_{\mathrm{f}}$ of TNS in the water pool of the microemulsions compared to ordinary bulk water indicates that the microenvironment of the TNS in the water pool is significantly different from bulk water. On further addition of water as the water pool swells in size and its polarity increas$\mathrm{es}^{26-28}$ the $\phi_{\mathrm{f}}$ decreases gradually to 0.063 at $w_{0}=32$ and $\lambda_{\max }^{\mathrm{em}}$ exhibits a red shift to $450 \mathrm{~nm}$. However it should be noted that even at $w_{0}=32$ emission properties of TNS is markedly different from those in bulk water. The emission properties of TNS in AOT/heptane/water microemulsions are summarized in Table 1.

\section{Time resolved studies}

Time resolved emission studies indicate that while in aqueous solution lifetime of TNS is extremely short $(\approx 80 \mathrm{ps})^{32}$, in microemulsions the fluorescence decay of TNS becomes much longer (Fig. 2 and Table 1). Obviously extracting meaningful rate constants in such a heterogeneous system is difficult. ${ }^{29,30}$ Inside the water pool the polarity of the different layers of water depends quite strongly on their distance from the ionic headgroup of AOT. Robinson et al. pointed out that if the diffusion coefficient $(D)$ of the probe in the water pool is same as that of an aromatic molecule in ordinary water $\left(0.05 \AA^{2} \mathrm{ps}^{-1}\right)$, the probe molecule moves about $10 \AA$ per ns in a direction normal to the surface $\left.\left(<z^{2}\right\rangle=2 D<t>\right) .{ }^{4}$ Thus the probe, TNS, molecules pass through several water layers of different polarity

Table 1 Emission characteristics of TNS in 0.09 M AOT in heptane

\begin{tabular}{rrrcc}
\hline$w_{0}$ & \multicolumn{1}{c}{$\phi_{\mathrm{f}}$} & $\tau_{\mathrm{f}} / \mathrm{ns}$ & $k_{\mathrm{r}}\left(\times 10^{8}\right) / \mathrm{s}^{-1}$ & $k_{\mathrm{nr}}\left(\times 10^{8}\right) / \mathrm{s}^{-1}$ \\
\hline 4 & 0.29 & 4.0 & 0.725 & 1.775 \\
8 & 0.125 & 1.77 & 0.706 & 4.94 \\
12 & 0.094 & 1.30 & 0.723 & 6.97 \\
32 & 0.063 & 0.95 & 0.663 & 9.86 \\
\hline
\end{tabular}


within the observed lifetime of a few ns. In such a situation one often encounters a multiexponential decay which originates from the different polarity regions experienced by the probe molecule within its lifetime. If the diffusion coefficient inside the water pool is slower by one order of magnitude compared to ordinary aqueous solution, every TNS molecule would be more or less confined to one layer of water molecules. However, even then there is possibility of different TNS molecules in different polarity regions being excited simultaneously. Instead of giving too much importance on the magnitude of individual decay constants of such multiexponential decays in such a complex situation we have fitted the decay to a single exponential to get an average picture. From the observed $\phi_{\mathrm{f}}$ and lifetime $\left(\tau_{\mathrm{f}}\right)$, radiative $\left(k_{\mathrm{r}}\right)$ and nonradiative rates $\left(k_{\mathrm{nr}}\right)$ of TNS in AOT/heptane/water microemulsion have been calculated as explained elsewhere. ${ }^{19}$ The results summarized in Table 1 indicates that with increase in the water content the lifetime of TNS in the microemulsion decreases from $4 \mathrm{~ns}$ at $w_{0}=4$ to $0.95 \mathrm{~ns}$ at $w_{0}=32$ and the nonradiative TICT rate increases from $1.775 \times 10^{8} \mathrm{~s}^{-1}$ at $w_{0}=4$ to $9.86 \times 10^{8} \mathrm{~s}^{-1}$ at $w_{0}=32$. It may be noted that compared to ordinary bulk water in the water pool at $w_{0}=4$ the lifetime of TNS increases about 50 times while the TICT rate decreases about 70 times. It may be noted such a nanosecond component in the fluorescence lifetime of a closely related molecule 1,8anilinonaphthalene sulfonate has also been reported at the heptane-water interface by Teramae et al. ${ }^{2}$ and in the microemulsions by Robinson et al. ${ }^{4}$

Compared to bulk water the solvation dynamics slows down several thousand times inside the reverse micelles. ${ }^{7-9}$ The interior of the water pools differs in two ways from ordinary bulk water. Firstly, the dielectric relaxation time $\left(\tau_{\mathrm{D}}\right)$ indicates a bimodal behavior with one component of 10 ps which is similar to ordinary water and another of $10 \mathrm{~ns}$ which is thousand times slower compared to bulk water. ${ }^{11-14}$ Secondly, comparing the position of the steady state absorption or emission maxima of many probe molecules in the water pools with those in different solvents ${ }^{26-28}$ (or comparing present data on TNS with ref.19) the static dielectric constant $\left(\varepsilon_{0}\right)$ of the water pool of the microemulsions seems to be close to that of alcohol (i.e. $\varepsilon_{0} \approx 30$ ). The solvation dynamics in a medium is controlled by the longitudinal relaxation time, $\tau_{\mathrm{L}}=\left(\varepsilon_{\infty} / \varepsilon_{0}\right) \tau_{\mathrm{D}}$, where $\varepsilon_{\infty}$ and $\varepsilon_{\mathrm{o}}$ are respectively the high frequency and static dielectric constants and $\tau_{\mathrm{D}}$, the dielectric relaxation time of the medium. ${ }^{5,6}$ If one makes the assumption that $\varepsilon_{\infty}$ inside the water pool is same as that of ordinary water (i.e. 5) one immediately expects a component of $(5 / 30) \times \tau_{\mathrm{D}}$. This leads to solvation times of $1.6 \mathrm{ps}$ and $1.6 \mathrm{~ns}$ corresponding to the $10 \mathrm{ps}$ and $10 \mathrm{~ns}$ dielectric relaxation time respectively. Thus the dramatic slowing down of the solvation dynamics inside the water pool corresponds to the $1.6 \mathrm{~ns}$ solvation time inside the water pool and is due to the thousand fold decrease in the dielectric relaxation time of water in organized media. The ICT process is, however, governed by the static polarity of the medium as discussed by Eisenthal et $a l .{ }^{17}$ In this case the solvent affects the dynamics of the ICT process by modifying the activation barrier for the ICT process due to the differential stabilization of the "nonpolar" reactant and the polar product (TICT). This causes a decrease in the activation barrier of the twisted ICT process with rise in polarity of the medium. The retardation of the ICT process of TNS inside the water pool is, thus, ascribed to the lower static polarity of the water pool compared to bulk water.

In summary the present work demonstrates that the dynamics of the ICT process of TNS inside the water pool of AOT/heptane microemulsions are slower by a factor of $\approx 70$ at $w_{0}=4$ (which corresponds to $r_{\mathrm{w}}=8 \AA$ ) and by a factor of $\approx 12.7$ at $w_{0}=32\left(r_{\mathrm{w}}=64 \AA\right)$ compared to bulk water. The retardation of the ICT process is attributed to the lower static polarity of the water pool compared to bulk water. While dielectric relaxation behavior of the water molecules in such systems reveals a component thousand times slower compared to bulk water, the static dielectric constant decreases only about two times. Since the two fold decrease in the static dielectric constant corresponds to a small decrease in the polarity of the medium (i.e. $E_{\mathrm{T}}(30)$ values) from 63.6 in bulk water to about 50 in microemulsions and since the rate of the ICT process decreases exponentially with $E_{\mathrm{T}}(30), 12-70$ fold decrease in the rate of the ICT process is observed.

Thanks are due to the Department of Science and Technology and Council of Scientific and Industrial Research (CSIR), Government of India for generous research grants. CSIR is also thanked for providing fellowship to A.D., D.M. and S.K.P.

\section{References}

1. K. B. Eisenthal, Chem. Rev., 96, 1343 (1996).

2. K. Bessho, T. Uchida, A. Yamauchi, T. Shiyoa and N. Teramae, Chem. Phys. Lett., 264, 381 (1997).

3. M. Yanagimachi, N. Tamai and H. Masuhara, Chem. Phys. Lett., 100, 469 (1992).

4. C. H. Cho, M. Chung, J. Lee, T. Nguyen, S. Singh, M. Vedamuthu, S. Yao, S.-B. Zhu and G. W. Robinson, J. Phys. Chem., 99, 7806 (1995).

5. S. Vajda, R. Jimenez, S. Rosenthal, V. Fiddler, G. R. Fleming and E. Castner. Jr., J. Chem. Soc. Faraday Trans., 91, 867 (1995).

6. N. Nandi and B. Bagchi, J. Phys. Chem., 100, 13914 (1996).

7. J. S. Lundgren, M. P. Heitz and F. V. Bright, Anal. Chem., 67, 3775 (1995)

8. S. Das, A. Datta and K. Bhattacharyya, J. Phys. Chem., A101, 3299 (1997).

9. N. Sarkar, K. Das, A. Datta, S. Das and K. Bhattacharyya, J. Phys. Chem., 100, 10523 (1996).

10. N. Sarkar, A. Datta, S. Das and K. Bhattacharyya, J. Phys. Chem., 100, 15483 (1996).

11. D. W. Urry, S. Peng, J. Xu and D. T. McPherson, J. Am. 
Chem. Soc., 119, 1161 (1997).

12. P. S. Belton, J. Phys. Chem., 99, 17061 (1995).

13. V. P. Denisov and B. Halle, J. Am. Chem. Soc., 116, 10324 (1994).

14. M. Fukazaki, N. Miura, N. Shiyasiki, D. Kuita, S. Shiyoya, M. Haida and S. Mashimo, J. Phys. Chem., 99, 431 (1995).

15. Z. R. Grabowski, Pure Appl. Chem., 65, 1751 (1993).

16. K. Bhattacharyya and M. Chowdhury, Chem. Rev., 93, 507 (1993).

17. J. M. Hicks, M. Vandersall, Z. Babarogic and K. B. Eisenthal, Chem. Phys. Lett., 116, 18 (1985).

18. T. L. Chang and H. C. Cheung, Chem. Phys. Lett., 173, 343 (1990).

19. K. Das, N. Sarkar, D. Nath and K. Bhattacharyya, Spectrochim. Acta, 48A, 1701 (1992).

20. W. O. McClure and G. M. Edelman, Biochemistry, 5, 1908 (1966).

21. P. L. Luisi, Angew. Chem. Int. Ed. Engl., 24, 439 (1985).

22. T. K. Jain, M. Varshney and A. Maitra, J. Phys. Chem., 93, 7409 (1989).

23. A. Maitra, J. Phys. Chem., 88, 5122 (1984).

24. K. Mukherjee, D. C. Mukherjee and S. P. Moulik,

\section{Langmuir, 9, 1727 (1993).}

25. J. Eastoe, W. K. Young and B. H. Robinson, J. Chem. Soc. Faraday Trans., 86, 2883 (1990).

26. M. Wong, J. K. Thomas and M. Gratzel, J. Am. Chem. Soc., 98, 2391 (1976).

27. M. Belletete, M. Lachapelle and G. Durocher, J. Phys. Chem., 95, 5337 (1990)

28. J. Guha Ray and P. K. Sengupta, Chem. Phys. Lett., 75, 230 (1994).

29. D. Toptygin, J. Svobodova, I. Konopasek and L. Brand, J. Chem. Phys., 96, 7919 (1992).

30. D. R. James and W. R. Ware, Chem. Phys. Lett., 120, 485 (1985).

31. A. Datta, D. Mandal, S. K. Pal and K. Bhattacharyya, Chem. Phys. Lett., in press.

32. N. Sarkar, K. Das, S. Das, A. Datta, R. Datta and K. Bhattacharyya, J. Chem. Soc. Faraday Trans., 92, 3097 (1996).

(Received September 30, 1997)

(Accepted December 4, 1997) 